\title{
MODULI OF SEMISTABLE LOGARITHMIC CONNECTIONS
}

\author{
NITIN NITSURE
}

\section{INTRODUCTION}

Under the Riemann Hilbert correspondence, which is an equivalence of categories, local systems on a nonsingular complex projective variety $X$ correspond to pairs $E=(\mathscr{E}, \nabla)$ where $\mathscr{E}$ is a locally free sheaf on $X$, and $\nabla: \mathscr{E} \rightarrow \Omega_{X}^{1} \otimes \mathscr{E}$ is an integrable connection on $\mathscr{E}$. Generalizing this correspondence to noncomplete quasi-projective varieties, Deligne (see [D]) showed that the correct algebro-geometric objects which correspond to local systems on such a variety $Y$ are the so-called regular connections on $Y$. The condition of regularity on algebraic connections on $Y$ can be expressed as follows. Fix a Hironaka completion $X$ of $Y$, that is, nonsingular projective variety $X$ which contains $Y$ as an open subvariety, such that the complement $S=X-Y$ is a smooth divisor with normal crossings. A logarithmic connection $F=(\mathscr{F}, \nabla)$ on $X$ with singularities over $S$ is by definition a torsion-free coherent sheaf $\mathscr{F}$ on $X$ together with a map $\nabla: \mathscr{F} \rightarrow \Omega_{X}^{1}[\log S] \otimes \mathscr{F}$ which satisfies C-linearity and the Leibniz rule, where $\Omega_{X}^{1}[\log S]$ is the sheaf of rational 1-forms on $X$ with logarithmic poles on $S$. The curvature of the connection $\nabla$ is assumed to be zero. Finally, an algebraic connection $E=(\mathscr{E}, \nabla)$ on $Y$ is regular if and only if there exists a logarithmic connection $F$ on $X$ with singularities over $S$ which extends $E$. If such an extension $F$ exists, the underlying sheaf $\mathscr{F}$ can be chosen to be locally free, and will be called a logarithmic lattice for $E$. A logarithmic lattice for a given regular connection is of course not unique, but there is a canonical way of choosing it called Deligne's construction, which only depends on the choice of a set-theoretic section of the map exp: $\mathbf{C} \rightarrow \mathbf{C}^{*}$. As such a section is not even continuous, the Deligne lattice does not behave well in families of regular connections.

Simpson has given in [S] a construction of a moduli scheme for (nonsingular) connections on a projective variety $X$. However, a simple example shows that it would be unreasonable to expect in general the existence of a moduli scheme for regular connections on a quasi-projective variety $Y$. For this, let $Y$ be the affine line minus the origin, with coordinate $x$. The differential equation $d y / d x-t y / x=0$ with parameter $t$ defines a family of regular connections on the sheaf $\mathscr{O}_{Y}$, parametrized by the affine line $T$ with coordinate $t$. We can see that $t_{1}$ and $t_{2}$ parametrize isomorphic connections if and only if $t_{1}-t_{2}$

Received by the editors April 10, 1991 and, in revised form, May 28, 1992.

1991 Mathematics Subject Classification. Primary 14D20; Secondary 32C38, 14F05.

Partially supported by NSF grant no. DMS-8610730. 
is an integer. This shows that isomorphism classes are bad subsets (bad in the algebraic category) of the parameter scheme $T$. Therefore, to have a reasonable moduli problem, we take a Hironaka completion $X$ of $Y$, and consider the moduli problem for logarithmic connections on $X$ with singularities on $X-Y$.

The basic difference between the case of nonsingular connections treated in [S] and the case of logarithmic connections is that we have to make a definition of (semi)stability for logarithmic connections (see $\S 2)$ and restrict ourselves to these. These conditions are Zariski open conditions on parameter schemes of families, so not too many logarithmic connections are lost. Also, though (semi)stability depends on the choice of a polarization $\mathscr{O}_{X}(1)$ on $X$, a logarithmic connection with irreducible monodromy is always stable. On the contrary, as we show by examples in $\S 2$, a stable logarithmic connection does not necessarily have an irreducible monodromy. Such a notion of (semi)stability was not necessary in the nonsingular case, for in the course of Simpson's construction of moduli, all nonsingular connections correspond to semistable points in the sense of geometric invariant theory. Moreover, in the nonsingular case, stability in the sense of geometric invariant theory exactly corresponds to irreducibility of the monodromy.

We now describe the results contained in this paper. Section 2 contains a development of some basic properties of families of logarithmic connections. These properties are necessary for a systematic treatment of the moduli problem, and with appropriate changes, will also hold in the nonsingular case. In $\S 3$, we generalize Simpson's construction and obtain a moduli for ( $S$-equivalence classes of) semistable logarithmic connections. While the proof follows the pattern of Simpson, we had to overcome difficulties of a technical nature in the geometric invariant theoretic part of the construction in the logarithmic case, as semistability becomes a nontrivial condition. In $\S 4$, we prove that the tangent space to the moduli of logarithmic connections at a stable locally free logarithmic connection $E$ is canonically isomorphic to the first hypercohomology of the logarithmic de Rham complex associated to $\operatorname{End}(E)$. This theorem, with obvious changes, will also hold in the nonsingular case. Finally, in $\S 5$, we go back to regular connections and prove a rigidity theorem (local uniqueness) for logarithmic lattices. This theorem says that for any regular connection on $Y$, those logarithmic lattices on $X$ for which no two distinct eigenvalues of the residue differ by an integer are rigid. In particular, this shows that Deligne's construction gives a rigid lattice.

\section{FAMILIES OF SEMISTABLE LOGARITHMIC CONNECTIONS}

Let $X$ be a nonsingular complex projective variety together with an ample line bundle $L$, and let $S$ be a divisor on $X$ which is smooth with normal crossings. Let $\mathscr{D}_{X}$ be the sheaf of algebraic linear differential operators on $X$ and let $\mathscr{D}_{X}[\log S] \subset \mathscr{D}_{X}$ be the sub $\mathscr{O}_{X}$-algebra generated by germs of tangent vector fields on $X$ which preserve the ideal sheaf of the reduced subscheme $S$. In these terms, a logarithmic connection can be defined as follows, as the curvature of the connection is assumed to be zero.

Definition 2.1. A logarithmic connection on $X$ with singularities over $S$ is a $\mathscr{D}_{X}[\log S]$ module which is coherent and torsion free as an $\mathscr{O}_{X}$ module. 
Also note that a nonsingular connection on $X$ is simply a $\mathscr{D}_{X}$ module which is coherent as an $\mathscr{O}_{X}$ module. Unlike logarithmic connections, nonsingular connections $E$ are automatically locally free and enjoy the property that any coherent subsheaf $F$ that is invariant under the connection is in fact a vector subbundle and satisfies the equality $p(F, n) / r(F)=p(E, n) / r(E)$ between "normalized" Hilbert polynomials. (Here, $p(\mathscr{F}, n)=\chi\left(X, \mathscr{F} \otimes L^{\otimes n}\right)$ denotes the Hilbert polynomial of any coherent sheaf $\mathscr{F}$ on $X$ with respect to the chosen ample line bundle $L$, and $r(\mathscr{F})$ denotes its leading coefficient, which equals $\operatorname{rank}(\mathscr{F}) / \operatorname{dim}(X)$ ! if $\mathscr{F}$ is locally free.) It is because of this equality that all nonsingular connections correspond to semistable points in the sense of geometric invariant theory in the course of Simpson's construction. In the case of logarithmic connections, we make the following definition.

Definition 2.2. A logarithmic connection $E$ is stable if for every $\mathscr{O}_{X}$-coherent $\mathscr{D}_{X}[\log S]$ submodule $F$ of $E$ with $0 \neq F \neq E$ we have $p(F, n) / r(F)<$ $p(E, n) / r(E)$ for all sufficiently large $n$. Similarly, we can define semistability by changing " $<$ " to " $\leq$ ". We can also define $\mu$-stability and $\mu$-semistability analogously.

Propositions 2.3. Let $E$ be a logarithmic connection of rank $r$ for which the monodromy representation of $\pi_{1}(X-S)$ in $\mathrm{GL}(r)$ is irreducible. Then $E$ is stable.

Proof. This follows easily as any $\mathscr{O}_{X}$-coherent $\mathscr{D}_{X}[\log S]$ submodule is either generically zero or generically equal to $E$.

Examples 2.4. The following examples show that the monodromy representation of a stable logarithmic connection need not be irreducible.

Example 2.4.1. Let $X$ be a curve of genus $\geq 1$, and let $\rho: \pi_{1}(X) \rightarrow \mathrm{GL}(2)$ be a representation that is neither irreducible nor completely reducible. Let $V$ be the corresponding local system on $X$ and $W \subset V$ be the rank 1 subsystem. Let $P$ be any point of $X$, and set $S=\{P\}$ and $Y=X-S$. Let $U$ be a simply connected small open neighbourhood of $P$ in euclidean topology and let $V_{U}=W_{U} \oplus K_{U}$ be a splitting of $V$ restricted to $U$. Let $E$ be the holomorphic vector bundle on $X$ which equals $\mathscr{O}_{Y}^{\text {an }} \otimes V$ outside $P$ and equals $\mathscr{O}_{U}^{\text {an }}(-m P) \otimes$ $W_{U} \oplus \mathscr{O}_{U}^{\mathrm{an}}(m P) \otimes K_{U}$ on $U$ where $m$ is any positive integer. Then it is easy to see that $E$ has a natural logarithmic connection which has the reducible monodromy $\rho$. Also note that $\operatorname{degree}(E)=0$ and the only nonzero flat proper subbundle of $E$ has degree $-m$ and hence $E$ is stable.

Example 2.4.2. Let $X=P^{1}$ and $S=\{0,1, \infty\}$. Let $A$ be any nondiagonalizable matrix in $\mathrm{GL}(2)$. Define $\rho$ such that the local monodromy around $0,1, \infty$ is $A, I, A^{-1}$ respectively. Then by a construction analogous to the above (where 1 plays the role of $P$ ), we get a stable logarithmic connection on $P^{1}$ whose monodromy is reducible.

Proposition 2.5. Let $E, F$ be stable logarithmic connection with the same Hilbert polynomial. Then any $\mathscr{D}_{X}[\log S]$-homomorphism from $E$ to $F$ is either zero or an isomorphism, and the only global $\mathscr{D}_{X}[\log S]$-endomorphisms of $E$ are the constant multiples of $1_{E}$. 
Proof. The first statement follows immediately from the definition of stability. Since the generalized eigen-sheaves of any endomorphism of $E$ will be $\mathscr{D}_{X}[\log S]$ invariant, we see that there can be only one eigenvalue. As a nilpotent endomorphism is zero by the first statement, the endomorphism has to be a scalar.

Definition 2.6. A family of logarithmic connections on $X$ parametrized by a scheme $T$ is a $\mathscr{D}_{X \times T / T}[\log S]$-module $E_{T}$ on $E \times T$ which is $\mathscr{O}_{X \times T}$-coherent and flat over $T$ such that for any $t \in T$, its restriction $E_{t}$ to the scheme $X \times t$ is torsion free. We say that two families $E_{T}$ and $F_{T}$ parametrized by $T$ are equivalent if there exists an open cover of $T$ such that the pullbacks of the two families to this open cover are isomorphic.

Lemma 2.7 (Coherence and representability of integrable direct image). Let $E_{T}$ be a family of logarithmic connections parametrized by a scheme $T$ (which is locally noetherian and of finite type over the field of complete numbers).

(i) The sheaf $\left(\pi_{T}\right)_{*}\left(E_{T}^{\nabla}\right)$ is a coherent sheaf of $\mathscr{O}_{T}$ modules, where $E_{T}^{\nabla} \subset E_{T}$ is the kernel sheaf of $\nabla_{T}: E_{T} \rightarrow \Omega_{X \times T / T}^{1}[\log S] \otimes E_{T}$.

(ii) Consider the contravariant functor from schemes over $T$ to the fibered category of coherent sheaves on such schemes, which associates to $T^{\prime} \rightarrow T$ the sheaf $\left(\pi_{T^{\prime}}\right)_{*}\left(E_{T^{\prime}}^{\nabla}\right)$ on $T^{\prime}$ (which is coherent by $(\mathrm{i})$ above) where $\left(E_{T^{\prime}}, \nabla_{T^{\prime}}\right)$ is the pullback. Then there exists a linear scheme $V \rightarrow T$ which represents this functor.

Proof. The problem is clearly local over the base, so assume that $T=\operatorname{Spec} A$ for some affine algebra $A$. Let $\mathscr{U}$ be an affine open cover of $X$, and let $C_{i}^{\cdot}, C_{2}^{\cdot}$ be the Čech cochain complexes for $E_{T}$ and $\Omega_{X \times T / T}^{1}[\log S] \otimes E_{T}$ with respect to the open cover $\mathscr{U} \times \operatorname{Spec} A$. These are complexes of flat $A$-modules with finitely generated cohomologies, and $\nabla_{T}$ induces an $A$-linear cochain map $\delta: C_{1}^{\cdot} \rightarrow C_{2}^{\cdot}$. Now by an argument similar to the proof of the main lemma in the proof of the semicontinuity theorem, there exist bounded, positive complexes $L_{1}, L_{2}^{\cdot}$ of finitely generated projective $A$-modules with chain maps $\alpha: L_{1} \rightarrow$ $C_{1}^{\cdot}, \beta: L_{2}^{\cdot} \rightarrow C_{2}^{\cdot}$ which are quasi-isomorphisms under every base change and a chain map $\gamma: L_{1}^{\cdot} \rightarrow L_{2}^{\cdot}$ such that $\delta \circ \alpha=\beta \circ \gamma: L_{1}^{\cdot} \rightarrow C_{2}^{\cdot}$. By construction, $\left(\pi_{T}\right)_{*}\left(E_{T}^{\nabla}\right)$ is isomorphic to the kernel sheaf of $\left(\partial_{L_{1}}^{0}, \gamma^{0}\right): L_{1}^{0} \rightarrow L_{1}^{1} \oplus L_{2}^{0}$, and hence is coherent. On the other hand, the geometric kernel of the vector bundle morphism $\left(\partial_{L_{1}}^{0}, \gamma^{0}\right): L_{1}^{0} \rightarrow L_{1}^{1} \oplus L_{2}^{0}$ is the linear scheme over $\operatorname{Spec} A$ which represents the given functor.

Proposition 2.8. Let $E_{T}$ and $F_{T}$ be two families of stable logarithmic connections on $X$ with the same Hilbert polynomial, parametrized by a scheme $T$. Consider the following conditions.

(i) The families $E_{T}$ and $F_{T}$ are equivalent.

(ii) There exists a line bundle $J$ on $T$ such that $F_{T}$ is isomorphic to $E_{T} \otimes$ $\pi_{T}^{*} J$.

(iii) The sheaf $J=\left(\pi_{T}\right)_{*}\left(\operatorname{Hom}_{\mathscr{O}_{X \times T}}\left(E_{T}, F_{T}\right)^{\nabla}\right)$ on $T$ is invertible (where the 
$\mathscr{O}_{X \times T^{-m o d u l e}} \operatorname{Hom}_{\mathscr{O}_{X \times T}}\left(E_{T}, F_{T}\right)$ is given the induced logarithmic connection), and the natural map $E_{T} \otimes \pi_{T}^{*} J \rightarrow F_{T}$ is an isomorphism of $\mathscr{D}_{X \times T / T}[\log S]$ modules.

(iv) For each $t \in T$ the restrictions $E_{t}$ and $F_{t}$ to $X \times\{t\}$ are isomorphic logarithmic connections.

Then conditions (i), (ii), and (iii) are equivalent, and they imply (iv). Moreover, if the scheme $T$ is reduced then (iv) is equivalent to the others.

Proof. This follows by applying Lemma 2.7 to $\operatorname{Hom}_{\mathscr{O}_{X \times T}}\left(E_{T}, F_{T}\right)$ and using Proposition 2.5 and the following elementary lemma.

Lemma 2.9. Let $A$ be a noetherian ring, $M$ and $N$ finitely generated free $A$ modules, $\phi: M \rightarrow N$ a homomorphism, such that for each maximal ideal $\mathfrak{m} \subset A$, the kernel of the induced map $\phi_{\mathfrak{m}}: M / \mathfrak{m} M \rightarrow N / \mathfrak{m} N$ is a 1-dimensional vector space over $A / \mathfrak{m} A$. Moreover, suppose that there exists an element $x$ of kernel $\phi$, such that its image generates $\operatorname{ker} \phi_{\mathfrak{m}}$ for all $\mathfrak{m}$. Then $\operatorname{ker} \phi$ is a free $A$ module of rank 1 (with $x$ as a basis).

Remark. If the ring $A$ has Jacobson radical zero, then we do not need the help of $x$ for the conclusion to hold.

Lemma 2.10. Let $T$ be any scheme, and $m$ an integer.

(i) Let $\mathscr{F}$ be a coherent sheaf on $T$ such that any closed point $t \rightarrow T$, the pullback of $\mathscr{F}$ has dimension at most $m$ as a vector space over $k(t)$. Then there exists a unique closed subscheme $T_{0}$ of $T$ which has the following universal property. Any morphism $f: S \rightarrow T$ of schemes factors through $T_{0}$ if and only if the pullback $f^{*} \mathscr{F}$ is a locally free sheaf of rank $m$.

(ii) Let $\phi: E \rightarrow F$ be a morphism between locally free sheaves $E$ and $F$ on $T$ such that at any closed point $t \rightarrow T$, the kernel of the pullback $\phi_{(t)}: E_{t} \otimes k(t) \rightarrow$ $F_{t} \otimes k(t)$ is of dimension at most $m$ over $k(t)$. Then there exists a unique closed subscheme $T_{0}$ of $T$ which has the following universal property. Any morphism $f: S \rightarrow T$ factors through $T_{0}$ if and only if the kernel of the pullback $f^{*}(\phi): f^{*} E \rightarrow f^{*} F$ is a locally free sheaf of $\operatorname{rank} m$.

Proof. Statement (i) is easy to prove, and (ii) follows by applying (i) to the cokernel sheaf of the transpose map $\phi^{*}: F^{*} \rightarrow E^{*}$ between the dual sheaves.

Proposition 2.11. Let $E_{T}$ and $F_{T}$ be two families of stable logarithmic connections with the same Hilbert polynomial that are parametrized by a scheme $T$. Then $T$ has a closed subscheme $T_{0}$ which has the following property. A morphism $f: U \rightarrow T$ of schemes factors through $T_{0}$ if and only if the pullbacks $f^{*} E_{T}$ and $f^{*} F_{T}$ are equivalent families on $U$. In particular the underlying set of $T_{0}$, which consists of all $t \in T$ such that the restrictions of $E_{T}$ and $F_{T}$ to $X \times t$ are isomorphic, is closed in $T$.

Proof. The problem is clearly local over $T$ so we may assume that $T$ is affine. Let $G_{T}=\operatorname{Hom}_{\mathscr{O}_{X \times T}}\left(E_{T}, F_{T}\right)$ with the induced logarithmic connection. Taking $G_{T}$ in the place of the $E_{T}$ in the proof of Lemma 2.7, let $\gamma: L_{1} \rightarrow L_{2}$ be the corresponding morphism of complexes. Consider the linear map $\left(\partial_{L_{1}}^{0}, \gamma^{0}\right): L_{1}^{0} \rightarrow L_{1}^{1} \oplus L_{2}^{0}$ between locally free sheaves on $T$. Let $T_{0}$ be 
the closed subscheme of $T$ defined by applying Lemma 2.10 (ii) to this map, taking $m=1$. It follows from its construction using Proposition 2.5 that $T_{0}$ has the required properties.

Proposition 2.12. For any semistable logarithmic connection $E$, there exists a filtration $0 \subset E_{1} \subset \cdots \subset E_{l}=E$ by $\mathscr{O}_{X}$-coherent $\mathscr{D}_{X}[\log S]$ submodules such that each successive quotient is a stable logarithmic connection and has the same normalized Hilbert polynomial as $E$. The corresponding graded $\mathscr{D}_{X}[\log S]$ module $\operatorname{gr}(E)$ is independent of the choice of such a filtration.

Proof. Using Proposition 2.5, this follows by applying the Jordan-Hölder theorem to the category of all semistable logarithmic connections which have the given Hilbert polynomial.

Definition 2.13. We say that two logarithmic connections $E$ and $F$ are Sequivalent if $\operatorname{gr}(E)$ is isomorphic to $\operatorname{gr}(F)$.

Proposition 2.14. In any family of semistable logarithmic connections, the $S$ equivalence classes are closed in the parameter scheme. For any semistable logarithmic connection $E$, there exists a family of semistable logarithmic connections parametrized by the affine line $A^{1}$, such that at the origin of $A^{1}$ the family restricts to $\operatorname{gr}(E)$ and at any other $t \in A^{1}, E_{t}$ is isomorphic to the pullback of $E$ under $X \times\{t\} \rightarrow X$.

Proof. See, for example, the proofs of the corresponding statements for semistable Hitchin pairs in $\S 4$ of [Ni].

Proposition 2.15 (Openness of (semi)stability). For any family $E_{T}$ of logarithmic connections, the subset $T^{\text {Stable }}=\left\{t \in T \mid E_{t}\right.$ is stable $\}$ is open in $T$. Similarly, $T^{\text {Semistable }}$ is open.

Proof (by the quot scheme method of Narasimhan and Ramanathan). Let $Q \rightarrow$ $T$ be the relative quot scheme which parametrizes all quotient $\mathscr{O}_{Y \times\{t\}}$ modules $E_{t} \rightarrow \mathscr{F}$ such that $p(\mathscr{F}, n) / r(\mathscr{F}) \leq p\left(E_{t}, n\right) / r\left(E_{t}\right)$ for various arbitrarily large integers $n$. Then $Q$ is proper over $T$ by Lemma 2.7 of [G]. The set of all points in $Q$ where the kernel of $E_{t} \rightarrow \mathscr{F}$ is $\mathscr{D}_{X}[\log S]$ invariant is closed in $Q$; hence the complement of its image in $T$ is open. A similar argument will show that semistability is also an open condition.

\section{Construction OF THE MODULI}

In this section, we construct a coarse moduli scheme for S-equivalence classes of semistable logarithmic connections which have a given Hilbert polynomial. For this, we follow with some modifications the method used by Simpson for nonsingular connections. We will omit the details wherever the argument is very similar to that in $\S 4$ of [S].

Proposition 3.1 (Boundedness of underlying sheaves). We fix the Hilbert polynomial $P$. Then there exists an integer $N$ which depends only on $P$, such that for any semistable logarithmic connection $E$ with Hilbert polynomial $P$ and for any integer $n \geq N$, the $\mathscr{O}_{X}$-module $E(n)=E \otimes L^{\otimes n}$ is generated by global sections and all its higher cohomologies are zero. As a consequence, for all $n \geq N$ 
the underlying $\mathscr{O}_{X}$-modules of semistable logarithmic connections with the given Hilbert polynomial $P$ occur as points of the quot scheme QUOT $(n)$ of quotients of $\mathscr{O}_{X}(-n)^{\oplus P(n)}$ which have Hilbert polynomial $P$.

Proof. The proof is an analogue of the proof of Lemma 4.2 of [S] with the following changes.

(i) Instead of using $\mathscr{D}_{X}$ with its usual filtration $F_{i}$, use $\mathscr{D}_{X}[\log S]$ with the induced filtration $F_{i}^{\prime}=F_{i} \cap \mathscr{D}_{X}[\log S]$.

(ii) In place of the tangent sheaf $T(X)$ use the sheaf $T(X)[\log S]=T(X) \cap$ $\mathscr{D}_{X}[\log S]$.

(iii) In place of the property of nonsingular connections that $p(F) / r(F)=$ $p(E) / r(E)$ for any $\mathscr{O}_{X}$-coherent $\mathscr{D}_{X}$ submodule $F \subset E$, use the definition of semistability.

Remark 3.2. The proof of the above proposition shows that there exists an integer $c$ which depends only on the rank $r$ such that for any semistable logarithmic connection $E$ of rank $r$ and any coherent subsheaf $\mathscr{F} \subset E$ we have $\mu(\mathscr{F})<\mu(E)+c$.

Proposition 3.3. Let $T$ be a scheme and let $\mathscr{E}$ be a coherent sheaf on $X \times T$ which is flat over $T$. Consider the functor on $T$-schemes which associates to $f: T^{\prime} \rightarrow T$ the set of all $\mathscr{D}_{X \times T^{\prime} / T^{\prime}}[\log S]$-module structures on $\left(1_{X} \times f\right)^{*} \mathscr{E}$. Then there exists a scheme $\mathscr{A}(\mathscr{E})$ of finite type over $T$ which represents this functor.

Proof. We first give a logarithmic version of Grothendieck's definition of a connection. Let the sheaf $R=\mathscr{O}_{X} \oplus \Omega_{X}^{1}[\log S]$ be given a ring structure by defining $(f, \alpha)(g, \beta)=(f g, f \beta+g \alpha)$. The projection $R \rightarrow \mathscr{O}_{X}$ is a ring homomorphism. In the reverse direction, we have two ring homomorphisms $\mathscr{O}_{X} \rightarrow R$ which are defined by $f \mapsto(f, 0)$ and $f \mapsto(f, d f)$ respectively. Let $\left.X_{1}[\log S]\right]=\operatorname{Spec} R$ (this scheme is the logarithmic version of the thickened diagonal $\left.X_{1} \hookrightarrow X \times X\right)$. Now, the above ring homomorphisms give a map $i: X \rightarrow X_{1}[\log S]$ and two maps $p_{1}, p_{2}: X_{1}[\log S] \rightarrow X$ with $p_{1} \circ i=p_{2} \circ i=1_{X}$. A logarithmic connection (with curvature not necessarily zero) on a sheaf $\mathscr{E}$ on $X$ is the same as an isomorphism $\phi: p_{1}^{*}(\mathscr{E}) \rightarrow p_{2}^{*}(\mathscr{E})$ over $X_{1}[\log S]$ such that $i^{*}(\phi)=1_{\mathscr{E}}$ on $X$. With this preparation, now the proof of the proposition follows from an argument similar to the proof of the corresponding statement for nonsingular connections in $\S 4$ of [S].

Now let $A_{n}^{\prime}$ be the scheme over QUOT $(n)$ constructed by applying Proposition 3.3 to the universal quotient sheaf. Let $A_{n}$ be the open subset consisting of all $s \in A_{n}^{\prime}$ such that for the corresponding $\mathscr{D}_{X}[\log S]$ module $E_{s}$,

(i) The map $H^{0}\left(X_{s}, \mathscr{O}_{X_{s}}\right)^{\oplus P(n)} \rightarrow H^{0}\left(X_{s}, E_{s}\right)$ is an isomorphism.

(ii) As an $\mathscr{O}_{X_{s}}$ module, $E_{s}$ is torsion free.

Let $A_{n}^{S}$ (respectively, $A_{n}^{S S}$ ) be the open subset of $A_{n}$ where $E_{s}$ is a stable (respectively, semistable) logarithmic connection. Then from the universal property of $A_{n}^{\prime}$ it follows that the action of $\operatorname{PGL}(P(n))$ on QUOT $(n)$ has a canonical lift to $A_{n}^{\prime}$. From the definitions of $A_{n}, A_{n}^{S}$, and $A_{n}^{S S}$ it follows that these 
are invariant under this action. It can now be seen that the following is true.

Proposition 3.4. The family parametrized by $A_{n}^{S S}$ has the local universal property for families of semistable logarithmic connections on $X$ with Hilbert polynomial $P$. The connections parametrized by two points of $A_{n}^{S S}$ are isomorphic if and only if they lie in the same $\operatorname{PGL}(P(n))$-orbit.

Construction of the quotient. In view of Propositions 2.14 and 3.4 above, if a good quotient $A_{n}^{S S} / \operatorname{PGL}(P(n))$ exists, it will be the coarse moduli scheme for S-equivalence classes of semistable logarithmic connections, and it will contain as an open subscheme the moduli $A_{n}^{S} / \operatorname{PGL}(P(n))$ for stable logarithmic connections. Our construction of this quotient has the following steps, which may be compared with $[\mathrm{S}]$ to see the changes that were necessary. We will omit the details of the steps when they do not differ much from [S].

Step 1. There exists an integer $n_{0}$ such that for any stable (resp. semistable) logarithmic connection $E$ with Hilbert polynomial $P$, any $\mathscr{O}_{X}$ coherent $\mathscr{D}_{X}[\log S]$ submodule $F \subset E$ and any $n \geq n_{0}$, we have $h^{0}(X, F(n)) / r(F)<$ $P(n) / r \quad$ (resp. $\quad h^{0}(X, F(n)) / r(F) \leq P(n) / r$, and if equality holds, then $p(F) / r(F)=P / r)$. In view of Remark 3.2 above, this follows by an argument similar to the first part of the proof of Theorem 2 of [S].

Step 2. Let $Q_{n}$ be the quot scheme of quotients $F_{r+1}^{\prime} \otimes \mathscr{O}_{X}(-n)^{P(n)} \rightarrow \mathscr{E}$ where $\mathscr{E}$ has Hilbert polynomial $P$. The right $\mathscr{O}_{X}$-module structure of $F_{r+1}^{\prime}$ is used for the tensor product. Let $U$ be the open subset of $Q$ where (i) the quotient sheaf $\mathscr{E}$ is torsion free, (ii) the composite map $\alpha: \mathscr{O}_{X}(-n)^{P(n)} \rightarrow F_{r+1}^{\prime} \otimes$ $\mathscr{O}_{X}(-n)^{P(n)} \rightarrow \mathscr{E}$ is surjective, and (iii) the induced map $H^{0}\left(X, \mathscr{O}_{X}\right)^{\oplus P(n)} \rightarrow$ $H^{0}(X, \mathscr{E}(n))$ is an isomorphism. The group $\operatorname{PGL}(P(n))$ acts on $Q_{n}$ and we have a canonical $\operatorname{PGL}(P(n))$-equivariant universally injective morphism of schemes $i: A_{n}^{S S} \rightarrow Q_{n}$ which factors through $U$.

Step 3. As in [S], the action of $\operatorname{SL}(P(n))$ on $Q_{n}$ has a sequence of linearizations $\psi_{m}$ indexed by sufficiently large integers $m$. Let $U^{S}$ and $U^{S S}$ denote the GIT-stable and GIT-semistable open subsets of $U$ with respect to the linearization $\psi_{m}$. Suppose $n$ is sufficiently large as above. Then under $i: A_{n} \rightarrow U$, the inverse images of $U^{S}$ and $U^{S S}$ are respectively equal to $A_{n}^{S}$ and $A_{n}^{S S}$ for all sufficiently large $m$. This follows from an argument similar to the proof of Lemma 4.5 of [S] with the following change: instead of the equality between the normalized Hilbert polynomials of any two nonsingular connections, use step 1 above.

Step 4. As stable logarithmic connections are mapped to GIT-stable points of $Q_{n}$, it can be seen by induction on the rank $r$ that polystable logarithmic connections are mapped to GIT-polystable points of $Q_{n}$ for large $n$, and for correspondingly large $m$. Hence if the closures in $Q_{n}^{S S}$ of the images of two orbits in $A_{n}^{S S}$ intersect then the closures of these two orbits must intersect in $A_{n}^{S S}$. This implies the following. Let $\phi: Q_{n}^{S S} \rightarrow N$ be the good quotient under $\operatorname{PGL}(P(n))$. Then the map $i: A_{n}^{S S} \rightarrow U^{S S}$ factors through the open subset 
$U^{\prime}=\phi^{-1}\left(N^{\prime}\right)$ of $U^{S S}$ where $N^{\prime}=N-\phi\left(Q_{n}^{S S}-U^{S S}\right)$.

Step 5. As the restriction $\phi: U^{\prime} \rightarrow N^{\prime}$ is a good quotient, it now suffices by Ramanathan's lemma (Proposition 3.12 of [Ne]) to show that the morphism $i: A_{n}^{S S} \rightarrow U^{\prime}$ is affine. Let $\pi: V \rightarrow U^{\prime}$ be the linear scheme over $U^{\prime}$ which represents the functor that associates to a scheme $T \rightarrow U^{\prime}$ over $U^{\prime}$ the $\Gamma\left(T, \mathscr{O}_{T}\right)$ module of all global sections over $X \times_{U^{\prime}} T$ of the pull-back of the sheaf $\mathscr{H}_{o m}\left(F_{r+1}^{\prime} \otimes \mathscr{E}, \mathscr{E}\right)$. Let $W$ be the closed subscheme of $V$ defined by the following two conditions. (i) At any $w \in W$ we must have $w \circ\left(1_{F_{r+1}^{\prime}} \otimes \alpha\right)=$ $\pi(w): F_{r+1}^{\prime} \otimes \mathscr{O}_{X}^{\oplus P(n)} \rightarrow \mathscr{E}$. (ii) Let $\mu: F_{1}^{\prime} \otimes F_{1}^{\prime} \rightarrow F_{2}^{\prime}$ denote the product, and for any $s \leq r+1$ let the induced map $F_{s}^{\prime} \otimes \mathscr{E} \rightarrow \mathscr{E}$ again be denoted by $w$. Then we must have $w \circ\left(1_{F_{1}^{\prime}} \otimes w\right)=w \circ\left(\mu \otimes 1_{\mathscr{E}}\right): F_{1}^{\prime} \otimes F_{1}^{\prime} \otimes \mathscr{E} \rightarrow \mathscr{E}$. Note that by construction, $W$ is affine over $U^{\prime}$. Also, the morphism $i: A_{n}^{S S} \rightarrow U^{\prime}$ factors through $W \rightarrow U^{\prime}$ giving an isomorphism $A_{n}^{S S} \rightarrow W$. This completes the proof of the following theorem.

Theorem 3.5. There exists a coarse moduli scheme $\mathscr{M}$ for S-equivalence classes of semistable logarithmic connections on $X$ with singularities over $S$, which have a given Hilbert polynomial $P$. The scheme $\mathscr{M}$ is separated, quasi-projective and has an open subscheme $\mathscr{M}^{\prime}$ which is the moduli for stable logarithmic connections with the given Hilbert polynomial.

\section{TANGENT SPACE TO THE MODULI}

An infinitesimal deformation of a logarithmic connection $E$ is a pair $\left(E_{V}, \alpha\right)$ where $E_{V}$ is a family of logarithmic connections parametrized by $V=$ $\operatorname{Spec} \mathrm{C}[\varepsilon] /\left(\varepsilon^{2}\right)$ and $\alpha$ is an isomorphism of $E_{V} / \varepsilon E_{V}$ with $E$. Two infinitesimal deformations $\left(E_{V}, \alpha\right)$ and $\left(F_{V}, \beta\right)$ will be called equivalent if there exists an isomorphism between $E_{V}$ and $F_{V}$ which carries $\alpha$ to $\beta$. By standard arguments of deformation theory, the set $T_{E}$ of all equivalence classes of infinitesimal deformations of $E$ has a natural structure of a vector space, and by the universal property of moduli, this is the tangent space to the moduli at the point $E$ when $E$ is stable. Note that when $E$ is locally free, the vector space $H^{1}(X, \operatorname{End}(\mathscr{E}))$ parametrizes the infinitesimal deformations of the underlying sheaf $\mathscr{E}$ of $\mathscr{O}_{X}$ modules, so there is a canonical linear map $q: T_{E} \rightarrow H^{1}(X, \operatorname{End}(\mathscr{E}))$. Let the sheaf $\mathscr{K}_{E}$ be the kernel of $\nabla: \Omega_{X}^{1}[\log S] \otimes$ $\operatorname{End}(\mathscr{E}) \rightarrow \Omega_{X}^{2}[\log S] \otimes \operatorname{End}(\mathscr{E})$. As the curvature of $\nabla$ is zero, the image of $\nabla: \operatorname{End}(\mathscr{E}) \rightarrow \Omega_{X}^{1}[\log S] \otimes \operatorname{End}(\mathscr{E})$ is contained in $\mathscr{K}_{E}$. If $A \in H^{0}\left(X, \mathscr{K}_{E}\right)$, then $\nabla+\varepsilon A$ is a family of logarithmic connections on the sheaf $\mathscr{E}$ parametrized by $V$. This association gives a linear map $p: H^{0}(X, \operatorname{End}(\mathscr{E})) \rightarrow T_{E}$.

Proposition 4.1. The following sequence of vector spaces is exact, where the maps $H^{i}(X, \operatorname{End}(\mathscr{E})) \rightarrow H^{i}\left(X, \mathscr{K}_{E}\right)$ are induced by $\nabla$.

$$
H^{0}(X, \operatorname{End}(\mathscr{E})) \rightarrow H^{0}\left(X, \mathscr{K}_{E}\right) \rightarrow T_{E} \rightarrow H^{1}(X, \operatorname{End}(\mathscr{E})) \rightarrow H^{1}\left(X, \mathscr{K}_{E}\right) .
$$

Proof. Any element $\alpha \in T_{E}$ can be described by an open covering $\mathscr{U}=\left(U_{i}\right)$ of $X$, a Čech co-cycle $\left(h_{i j}\right) \in Z^{1}(\mathscr{U}, \operatorname{End}(\mathscr{E}))$ and elements $A_{i} \in \Gamma\left(U_{i}, \mathscr{K}_{E}\right)$ 
such that $\left(\nabla+\varepsilon A_{i}\right)\left(1+\varepsilon h_{i j}\right)=\left(1+\varepsilon h_{i j}\right)\left(\nabla+\varepsilon A_{j}\right)$ on $U_{i} \cap U_{j}$, where $\varepsilon^{2}=0$. This equation can be written as $\nabla\left(h_{i j}\right)=A_{j}-A_{i}$. Note that $\left(h_{i j}\right)$ describes a deformation of $\mathscr{E}$ and $\left(A_{i}\right)$ describes a compatible deformation of $\nabla$, and together these describe the deformation $\alpha$ of $E$. From this description in terms of Čech cochains, the exactness of the 5-term sequence above follows.

Theorem 4.2. If a logarithmic connection $E$ is locally free, the vector space $T_{E}$ of infinitesimal deformations of $E$ (which equals the tangent space at $E$ to the moduli scheme of stable logarithmic connections when $E$ is stable) is canonically isomorphic to the first hypercohomology $\mathbf{H}^{1}\left(\mathscr{C}_{E}\right)$ of the complex $\left.\mathscr{C}_{E}=\left(\nabla: \operatorname{End}(\mathscr{E}) \rightarrow \mathscr{K}_{E}\right)\right)$, which is in turn equal to the first hypercohomology of the logarithmic de Rham complex $\left(\Omega_{X}^{\cdot}[\log S] \otimes_{\mathscr{O}_{X}} \operatorname{End}(\mathscr{E}), \nabla\right)$ associated with $\operatorname{End}(E)$.

Proof. Let $(R, \mathbf{m})$ be a local ring of finite type over $\mathbf{C}$ with $\mathbf{m}^{2}=0$. Let $V=\operatorname{Spec} R$ and $\left(E_{V}, \alpha\right)$ be an infinitesimal deformation of $E$ parametrized by $V$. Let $0 \rightarrow \operatorname{End}\left(\mathscr{E}_{V}\right) \rightarrow \mathbf{A}_{V}(\mathscr{E}) \rightarrow \pi_{V}^{*} T V \rightarrow 0$ be the relative Atiyah exact sequence on $X \times V$ for the family $\mathscr{E}_{V}$. Let $P$ be the closed point of $V$. The above sequence remains exact when restricted to $X \times\{P\} \hookrightarrow X \times V$. Consider the following push-out diagram, where $\mathbf{B}$ denotes the push-out term and $\mathbf{A}_{V}(\mathscr{E})_{P}$ denotes the restriction $\left.\mathbf{A}_{V}(\mathscr{E})\right|_{X \times\{P\}}$.

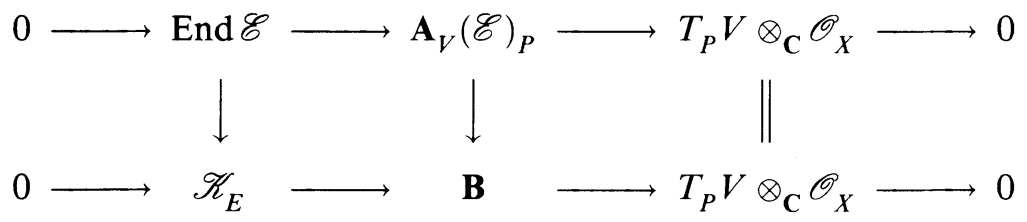

In the above diagram, the first vertical map is $\nabla$ and the last vertical map is identity. By Proposition 4.1 above, the composite map $T_{P} V \rightarrow H^{1}(X, \operatorname{End}(\mathscr{E}))$ $\rightarrow H^{1}\left(X, \mathscr{K}_{E}\right)$ is zero; hence the push-out is split. Hence, the map End $\mathscr{E} \rightarrow$ $\mathscr{K}_{E}$ has an extension to $\mathbf{A}_{V}(\mathscr{E})_{P}$. We now explicitly describe one extension, which will be canonical (in the sense described later). The deformation $\left(E_{V}, \alpha\right)$ can be described by an open covering $\mathscr{U}=\left(U_{i}\right)$ of $X$, a Čech co-cycle $\left(h_{i j}\right) \in$ $Z^{1}\left(\mathscr{U}, \operatorname{End}(\mathscr{E}) \otimes_{\mathbf{C}} \mathbf{m}\right)$ and elements $A_{i} \in \Gamma\left(U_{i}, \mathscr{K}_{E} \otimes_{\mathbf{C}} \mathbf{m}\right)$ such that $\left(\nabla+A_{i}\right)\left(1+h_{i j}\right)=\left(1+h_{i j}\right)\left(\nabla+A_{j}\right)$ on $U_{i} \cap U_{j}$. This equation can be written as $\nabla\left(h_{i j}\right)=A_{j}-A_{i}$. Note that $\left(h_{i j}\right)$ describes a deformation of $\mathscr{E}$ and $\left(A_{i}\right)$ describes a compatible deformation of $\nabla$, and together these describe the deformation $\left(E_{V}, \alpha\right)$ of $E$. Note that with respect to chosen local splittings as $\operatorname{End}(\mathscr{E}) \oplus\left(T_{P} V \otimes_{\mathbf{C}} \mathscr{O}_{X}\right)$ over the open sets $U_{i}$, the transition matrices $a_{i j}$ for $\mathbf{A}_{V}(\mathscr{E})_{P}$ have the form

$$
a_{i j}=\left(\begin{array}{cc}
1_{\text {End } \mathscr{E}} & h_{i j} \\
0 & 1_{T_{P} V \otimes_{\mathbf{C}} \mathscr{O}_{X}}
\end{array}\right) .
$$

We define a map $\eta_{\left(E_{V}, \alpha\right)}: \mathbf{A}_{V}(\mathscr{E})_{P} \rightarrow \mathscr{K}_{E}$ by the matrix $\psi_{i}=\left(\nabla, A_{i}\right)$ over $U_{i}$. These glue together as $\psi_{i} a_{i j}=\psi_{j}$ on $U_{i} \cap U_{j}$. The resulting map $\eta_{\left(E_{v^{\prime}}, \alpha\right)}$ 
can be seen to be independent of the local description (open cover and local trivializations) chosen. It obviously extends the map $\nabla: \operatorname{End}(\mathscr{E}) \rightarrow \mathscr{K}_{E}$, and it is canonical in the following sense. Given any other local algebra $\left(R^{\prime}, \mathbf{m}^{\prime}\right)$ with $\mathbf{m}^{\prime 2}=0$, and a local homomorphism $R \rightarrow R^{\prime}$, the map $\eta_{\left(E_{V}, \alpha\right)}$ pulls back to the corresponding map defined for the pullback of $\left(E_{V}, \alpha\right)$. Now consider the following short exact sequence of (vertical) complexes of sheaves on $X$. Note that the differentials in the complexes are only $\mathbf{C}$-linear.

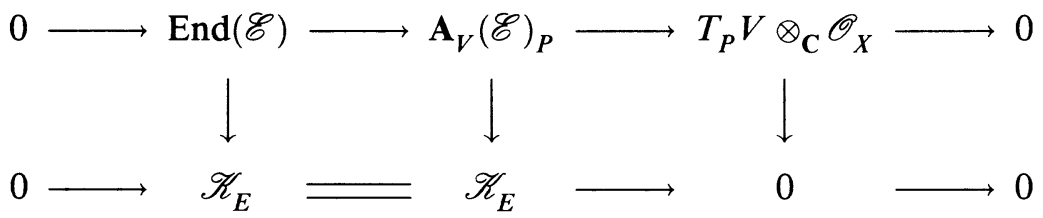

Let $\mathscr{C}_{E}$ denote the first vertical complex. Then taking hypercohomology, we get a functorial map $T_{P} V \rightarrow \mathbf{H}^{1}\left(\mathscr{C}_{E}\right)$. As $T_{E}$ is the direct limit of the $T_{P} V$, we get a canonical map $T_{E} \rightarrow \mathbf{H}^{1}\left(\mathscr{C}_{E}\right)$. With this map, the following diagram commutes, where the first row is the exact sequence of Proposition 4.1, and the second row is the small term exact sequence associated to hypercohomology.

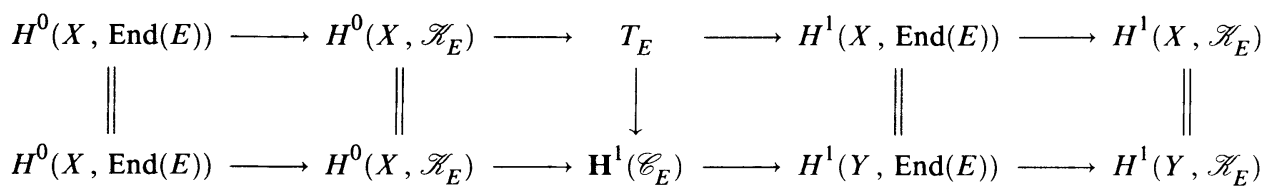

Hence the theorem follows from the five lemmas.

Remark 4.3. A similar argument will give another proof of the result of Biswas and Ramanan (which is a part of their forthcoming paper [B-R]) that for a Hitchin pair $(E, \phi: E \rightarrow L \otimes E)$ on a variety $X$, the space $T_{(E, \phi)}$ of infinitesimal deformations is canonically isomorphic to the first hypercohomology of the complex $[\phi,-]: \operatorname{End}(E) \rightarrow L \otimes \operatorname{End}(E)$.

\section{RIGIDITY OF LOGARITHMIC LATTICES FOR REGULAR CONNECTIONS}

Let $Y$ be a nonsingular quasi-projective variety which is not complete. Let $E$ be a connection on $Y$ (that is, $E$ is an $\mathscr{O}_{Y}$-coherent $\mathscr{D}_{Y}$-module). Recall that a Hironaka completion $X$ of $Y$ is a smooth projective variety which contains $Y$ as an open subset, such that $S=X-Y$ is a divisor which is smooth with normal crossings. Such a completion always exists, and any two such completions are dominated by a third such completion. We fix one such completion $X$.

Definition 5.1. Let $F$ be a $\mathscr{D}_{X}[\log S]$-submodule of $j_{*}(E)$ (where $j: Y \rightarrow X$ is the open inclusion) which is $\mathscr{O}_{X}$-coherent and locally free, and which restricts to $E$ on $Y$. Then $F$ will be called a logarithmic lattice for $E$ on $X$. A connection $E$ on $Y$ is regular if and only if on some (and, equivalently, on every) Hironaka completion $X$ of $Y$, there exists a logarithmic lattice $F$ for $E$.

Observe that by definition, a logarithmic lattice is a locally free logarithmic connection. 
The infinitesimal deformations of a connection $E$ on $Y$ form a vector space $T_{E}$ which, as in Proposition 4.1 above, fits in the following five term exact sequence where $\mathscr{K}_{E}$ is the kernel sheaf of $\nabla: \Omega_{Y}^{1} \otimes \operatorname{End}(\mathscr{E}) \rightarrow \Omega_{Y}^{2} \otimes \operatorname{End}(\mathscr{E})$.

$$
H^{0}(Y, \operatorname{End}(\mathscr{E})) \rightarrow H^{0}\left(Y, \mathscr{K}_{E}\right) \rightarrow T_{E} \rightarrow H^{1}(Y, \operatorname{End}(\mathscr{E})) \rightarrow H^{1}(Y, \mathscr{K}) .
$$

In fact, as in Theorem 4.2, $T_{E}$ is the hypercohomology of the corresponding complex $\nabla: \operatorname{End}(\mathscr{E}) \rightarrow \mathscr{K}_{E}$. Let $F$ be a logarithmic lattice for $E$ on $X$. Then restriction to $Y \hookrightarrow X$ gives a canonical linear map $T_{F} \rightarrow T_{E}$ between these two spaces of infinitesimal deformations.

Definition 5.2. Let $E$ be a regular connection on $Y$, and let $F$ be a logarithmic lattice for $E$ on $X$. We say that $F$ is rigid if the map $T_{F} \rightarrow T_{E}$ is injective.

Recall that to each logarithmic connection $F$, there corresponds an endomorphism $\operatorname{res}(F)$ of the pullback of the underlying $\mathscr{O}_{X}$-module $\mathscr{E}$ to the normalization $S^{*}$ of the divisor $S$, called the residue of $F$. The characteristic polynomial of $\operatorname{res}(F)$ has coefficients which are constant on each component of $S^{*}$. In particular, on each component of $S^{*}$ we can talk of the characteristic values of the residue (which are called the exponents of $F$ ).

Proposition 5.3 (Rigidity of lattice). Let $E$ be a regular connection on $Y$. Let $F$ be a logarithmic lattice for $E$ on $X$ which has the property that on any component of the normalization of $X-Y$, no two distinct exponents of $F$ differ by an integer. Then the map $T_{F} \rightarrow T_{E}$ is injective, that is, $F$ is rigid.

Proof. Consider the following commutative diagram, where the rows are exact.

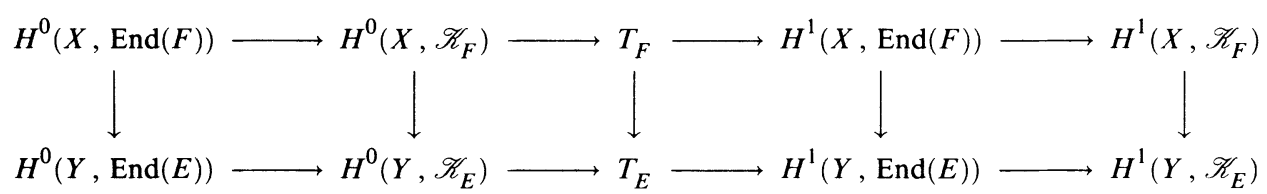

As before, any element $\theta \in T_{F}$ can be described by an open covering $\mathscr{U}=\left(U_{i}\right)$ of $X$, a Čech co-cycle $\left(h_{i j}\right) \in Z^{1}(\mathscr{U}, \operatorname{End}(\mathscr{F}))$ and elements $A_{i} \in \Gamma\left(U_{i}, \mathscr{K}_{F}\right)$ such that $\left(\nabla+\varepsilon A_{i}\right)\left(1+\varepsilon h_{i j}\right)=\left(1+\varepsilon h_{i j}\right)\left(\nabla+\varepsilon A_{j}\right)$ on $U_{i} \cap U_{j}$, where $\varepsilon^{2}=0$. This equation can be written as $\nabla\left(h_{i j}\right)=A_{j}-A_{i}$. Now suppose that $\theta \mapsto 0 \in T_{E}$. Then there exist $e_{i} \in \Gamma\left(U_{i} \cap Y, \operatorname{End}(\mathscr{E})\right)$ such that $h_{i j}=e_{j}-e_{i}$ on $U_{i} \cap U_{j} \cap Y$, and $\left(\nabla+\varepsilon A_{i}\right)\left(1+\varepsilon e_{i}\right)=\left(1+\varepsilon e_{i}\right) \nabla$ on $U_{i} \cap Y$. The last equality is equivalent to $\nabla\left(e_{i}\right)+A_{i}=0$. Note that the $\operatorname{End}(\mathscr{F})$ valued 1 -form $A_{i}$ has logarithmic singularities on $S$, while $\nabla$ is a logarithmic connection on $\operatorname{End}(\mathscr{F})$ with no nonzero integral exponents. Hence the equality $\nabla\left(e_{i}\right)=-A_{i}$ implies that the rational sections $e_{i}$ of $\operatorname{End}(\mathscr{F})$ on $U_{i}$ are in fact regular (holomorphic also on $S$ ). Now it can be seen that the original deformation $\theta$ is zero.

Remark 5.4. In particular, the logarithmic lattice associated to any regular connection by Deligne's construction is rigid.

\section{ACKNOWLEDGMENTS}

I am grateful to P. Deligne for useful conversations, and for pointing out some mistakes in the earlier version. I thank M. S. Narasimhan for a suggestion 
that was useful in proving Proposition 2.11, and S. Ramanan for telling me in advance about his result with Biswas on the tangent space to the moduli of Hitchin pairs, which inspired the corresponding result (Theorem 4.2) in $\S 4$. I thank I. Biswas and V. Srinivas for their remarks. I thank the Institute for Advanced Study, Princeton, for its hospitality while most of this work was done.

\section{REFERENCES}

[B-R] I. Biswas and S. Ramanan, An infinitesimal study of the moduli of Hitchin pairs. Preprint (1992) Tata Inst. Fund. Res., Bomday, India.

[D] P. Deligne, Equations différentielles à points singuliers réguliers, Lecture Notes in Math., vol. 163, Springer, Berlin-Heidelberg-New York, 1970.

[G] A. Grothendieck, Techniques de construction et théorèmes d'existence en géométrie algébrique. IV: Les schémas de Hilbert, Sem. Bourbaki exposé 221 (1960-61).

[Ne] P. E. Newstead, Introduction to moduli problems and orbit spaces, Tata Inst. Fund. Res. Lecture Notes, Springer, Berlin-Heidelberg-New York, 1978.

[Ni] N. Nitsure, Moduli space of semistable pairs on a curve, Proc. London Math. Soc. (3) 62 (1991), 275-300.

[S] C. Simpson, Moduli of representations of the fundamental group of a smooth projective variety, Preprint (1990), Princeton University.

School of Mathematics, Tata Institute of Fundamental Research, Homi Bhabha ROAD, BOMBAY 400 005, INDIA

E-mail address: nitsure@tifrvax.bitnet 